\title{
A de novo variant in OTX2 in a lamb with otocephaly
}

\author{
Julia Maria Paris', Anna Letko', Irene Monika Häfliger', Tanja Švara², Mitja Gombač², Primož Klinc³,

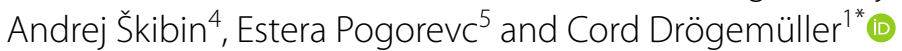

\begin{abstract}
Background: Otocephaly is a rare lethal malformation of the first branchial arch. While the knowledge on the causes of otocephaly in animals is limited, different syndromic forms in man are associated with variants of the PRRX1 and OTX2 genes.

Case presentation: A stillborn male lamb of the Istrian Pramenka sheep breed showed several congenital craniofacial anomalies including microstomia, agnathia, aglossia, and synotia. In addition, the lamb had a cleft palate, a small opening in the ventral neck region, a cystic oesophagus and two hepatic cysts. The brain was normally developed despite the deformed shape of the head. Taken together the findings led to a diagnosis of otocephaly. Whole-genome sequencing was performed from DNA of the affected lamb and both parents revealing a heterozygous single nucleotide variant in the OTX2 gene (Chr7: 71478714G > A). The variant was absent in both parents and therefore due to a de novo mutation event. It was a nonsense variant, XM_015097088.2:c.265C > T; which leads to an early premature stop codon and is predicted to truncate more than $70 \%$ of the OTX2 open reading frame (p.Arg89*).
\end{abstract}

Conclusions: The genetic findings were consistent with the diagnosis of the otocephaly and provide strong evidence that the identified loss-of-function variant is pathogenic due to OTX2 haploinsufficiency. The benefits of triobased whole-genome sequencing as an emerging tool in veterinary pathology to confirm diagnosis are highlighted.

Keywords: Agnathia, Domestic animal, Microstomia, Precision medicine, Rare disease, Synotia, Whole-genome sequencing

\section{Background}

Otocephaly is a rare severe malformation of the first branchial arch [1,2]. It is characterized by agnathia or mandibular hypoplasia, microstomia, aglossia or microglossia, and synotia and is often lethal [1-3]. Otocephaly is a key feature of two known rare disorders in man: agnathia-otocephaly complex (OMIM 202650) and microphthalmia with associated features including pituitary dysfunction (OMIM 610125). These mostly lethal phenotypes differ in severity and affected individuals

*Correspondence: cord.droegemueller@vetsuisse.unibe.ch

${ }^{1}$ Institute of Genetics, Vetsuisse Faculty, University of Bern, Bremgartenstr. 109a, 3001 Bern, Switzerland

Full list of author information is available at the end of the article rarely survive into childhood [4]. Two genes are known to be associated with these inherited disorders, the orthodenticle homeobox 2 (OTX2) and the paired related homeobox 1 (PRRX1) genes $[1,3,4]$. So far, all the OTX2related cases were heterozygous and mostly due to dominantly acting de novo variants, whereas the pathogenic variants described in the PRRX1 gene were either recessively or dominantly inherited [4]. Similar congenital anomalies including different syndromic forms of otocephaly occur also in domestic animals, for example in dogs (OMIA 001127-9615) [5], cattle (OMIA 0011279913), and sheep (OMIA 000023-9940) [6-9]. In a single case of ovine agnathia karyotyping revealed a diseasecausing reciprocal translocation [10]. So far, no single

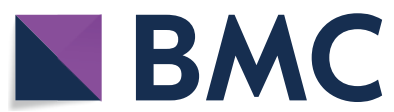

(c) The Author(s) 2020. This article is licensed under a Creative Commons Attribution 4.0 International License, which permits use, sharing, adaptation, distribution and reproduction in any medium or format, as long as you give appropriate credit to the original author(s) and the source, provide a link to the Creative Commons licence, and indicate if changes were made. The images or other third party material in this article are included in the article's Creative Commons licence, unless indicated otherwise in a credit line to the material. If material is not included in the article's Creative Commons licence and your intended use is not permitted by statutory regulation or exceeds the permitted use, you will need to obtain permission directly from the copyright holder. To view a copy of this licence, visit http://creativeco mmons.org/licenses/by/4.0/. The Creative Commons Public Domain Dedication waiver (http://creativecommons.org/publicdomain/ zero/1.0/) applies to the data made available in this article, unless otherwise stated in a credit line to the data. 
gene associated with otocephaly has been reported in any of these species.

\section{Case presentation}

In February 2018, an 8-year-old Istrian Pramenka ewe from a flock counting 500 sheep served by 16 different rams delivered a male lamb weighing $6 \mathrm{~kg}$ at term by caesarean section. The lamb died in less than a minute. At necropsy, severe craniofacial deformations were found. The lamb had dorso-ventrally deformed and shortened nasal region, very small oral aperture (microstomia), the tongue was missing (aglossia), and the hard palate was clefted (palatoschisis) (Fig. 1). The lower jaw was not found (agnathia). The auricular pinnae were located at the medioventral part of the head and neck (synotia) and without communication to the external auditory canal (meatus acusticus externus) (Fig. 1). Between the pinnae, there was an oval opening, measuring $1 \times 0.5 \mathrm{~cm}$, into a $2 \mathrm{~cm}$ long blind tubular structure. A later computed tomography (CT) scanning (Somatom Scope CT scanner, Siemens, Erlangen, Germany) of the head revealed deformed frontonasal bones and hyoid bones in abnormal position (Fig. 2). The oesophagus had a $1 \mathrm{~cm}$ long segmental stenosis and a proximal cystic dilatation. Additionally, two thin-walled cysts filled with yellow, translucent fluid were found on the surface of the liver. The brain and other organs were normally developed. The lungs had diffuse congenital atelectasis. Specimens of the brain, lung, heart, kidney, liver, adrenal gland, thymus and spleen were sampled for histopathology, fixed

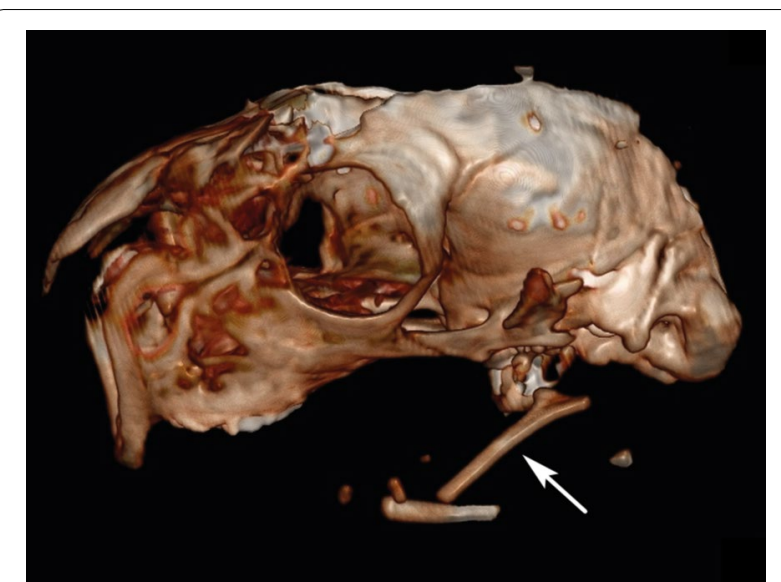

Fig. 2 Otocephaly in an Istrian Pramenka lambs-CT findings. Lateral CT scan of the head shows deformed frontonasal bones and displacement of the hyoid bones (arrow). The following CT parameters were used: $160 \mathrm{mAs}, 110 \mathrm{kV}, 1.0 \mathrm{~mm}$ slice thickness, $1.0 \mathrm{~mm}$ reconstruction increment and beam pitch 1.3

in $10 \%$ neutral buffered formalin, processed by routine methods, embedded in paraffin and sectioned at $4 \mu \mathrm{m}$ and stained with haematoxylin and eosin. Histopathological examination revealed normally developed organs, complete pulmonary atelectasis, mild generalised congestion, focal haemorrhages in the meninges and thymus and mild hydropic degeneration of the hepatocytes. The gross lesions and CT imaging findings were consistent with otocephaly.
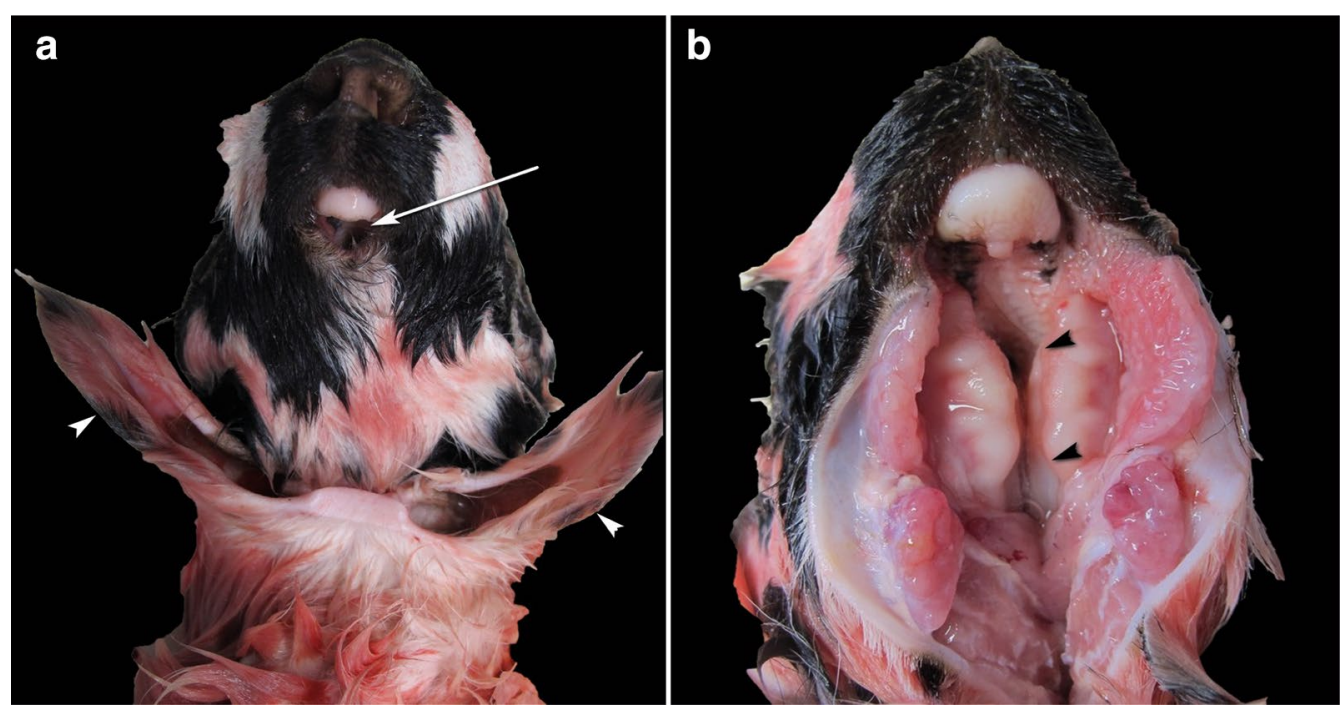

Fig. 1 Otocephaly in an Istrian Pramenka lamb_-gross pathology findings. a Ventral view of the head. The oral structures are very small (microstomia) (arrow) and the auricular pinnae (arrowheads) are located at the medioventral part of the head and neck (synotia). b Ventral view of the head with opened oral cavity. The palate is clefted (palatoschisis) (arrowheads) and the tongue is missing (aglossia). The lower jaw appears to be absent 
Genomic DNA of the lamb's skin and from EDTA blood of all sampled adults including the dam and 16 possible fathers was isolated using standard protocols. All 18 animals were genotyped for single nucleotide variants (SNV) on Illumina OvineSNP50 BeadChip array. PLINK v1.9 [11] was used for quality control of the data and parentage confirmation. Pruning based on missing genotype calls per marker (>10\%) resulted in 41,571 markers in the final dataset. Paternity status was checked for all 16 potential sires of the affected lamb and resulted in the exclusion of 15 rams and validation of a single ram as the sire. Subsequently, whole-genome sequencing of the affected lamb and both parents was carried out. Therefore, Illumina TruSeq PCR-free libraries were prepared for sequencing $2 \times 150$ bp paired-end reads on an Illumina HiSeq 3000 instrument. Read mapping to the Oar_rambouillet_v1.0 ovine reference genome assembly and variant calling and annotation was done as described previously [12]. The genotypes of the affected lamb were compared with both parents and 54 additional sheep genomes of various breeds that had been sequenced in the course of other ongoing studies (European Nucleotide Archive accession no. PRJEB30931). Due to the strong effect of the mutation, it was hypothesized that a loss-of-function variant affecting the coding sequence of a gene most likely would be responsible for the disease. In addition, it was hypothesized that the mutant allele of the causative variant should be completely absent from all other 54 sheep genomes in the control sample set. Adopting an assumption of a possible recessive mode of inheritance, 12 missense variants were found to be homozygous in the affected offspring and heterozygous in the parents (Additional file 1). Genome-wide filtering for proteinchanging sequence variants in the whole genome that were present heterozygous only in the affected lamb and homozygous wild type in the genomes of both parents, resulted in four variants representing putative de novo sequence variants (Additional file 1). The list of private protein-changing variants includes a de novo heterozygous nonsense variant in OTX2 (Chr7: 71478714G > A), a gene which is associated with otocephaly in people. The Integrative Genomics Viewer (IGV) was used for visual inspection of the identified variants [13] and confirmed that the variant was absent in both parents without any evidence for low-level mosaicism in any of the parents (Fig. 3a). Therefore, this variant was finally considered as the candidate causal mutation.

The variant was predicted to produce an early premature stop codon truncating more than $70 \%$ of the OTX2 open reading frame (p.Arg89*) and resulting in a significantly shorter protein lacking the $\mathrm{C}$-terminal OTX1-transcription factor binding domain of the protein (Fig. 3b). In comparison to the normal 289 amino acid-long OTX2 protein, also the homeobox domain of the protein is truncated (Fig. 3c), which alters this evolutionary highly conserved and functionally important protein region (Fig. 3d). Therefore, the OTX2: XM_015097088.2:c.265C > T variant most likely leads to a complete loss-of-function.

\section{Discussion and conclusions}

In the present study, a de novo nonsense variant in the ovine OTX2 gene was identified in a newborn lamb with otocephaly. The syndromic phenotype was characterized by microstomia, aglossia, palatoschisis, agnathia and synotia, and differed from previous similar case reports in sheep with synotia, otocephalus aprosopus or acephaly [7-9]. In a single stillborn lamb with splanchnocranial anomalies that were classified as aprosencephaly and otocephaly, an association with the OTX2 gene was ruled out [6]. Recently, morphological details of a single strophocephalic lamb with facial anomalies allocated to holoprosencephaly were presented but no molecular genetic examination was performed [14].

The reported OTX2 variant introduces an early stop codon into the open reading frame. Although the expression of the mutant OTX2 gene was experimentally analysed, it seems likely that the mutant transcripts will be subject to nonsense-mediated decay and that the OTX2:c. $265 \mathrm{C}>\mathrm{T}$ variant leads to a true null allele of the OTX2 gene. Due to the lack of appropriate material the RNA could not be analysed experimentally to confirm this hypothesis. The variant was absent in both parents and therefore due to a de novo mutation event. This mutation might have occurred during meiosis of parental, most likely paternal, germ cells and subsequently passed to the offspring as a consequence of low-level mosaicism in one of the parents. No evidence for further cases in the flock was obtained and therefore, the mutation might also have occurred post-zygotically during early embryonic development of the affected lamb explaining this single case.

The OTX2 homeobox gene plays a crucial role in craniofacial morphogenesis during early embryonic development. The OTX2 gene encodes a transcription factor, which is essential for the early development of the embryonic head and later also for the development of the eye and brain [4]. The OTX2 protein belongs to the OTX group of signalling molecules with a DNA-binding homeodomain region, which are highly conserved across all species [4]. Mouse models confirmed the involvement of OTX2 in pharyngeal arch formation and how its disruption leads to major craniofacial malformations [15].

On the basis of the combination of the available data, knowledge on OTX2 function in other species, and the fact that the identified nonsense variant is likely 


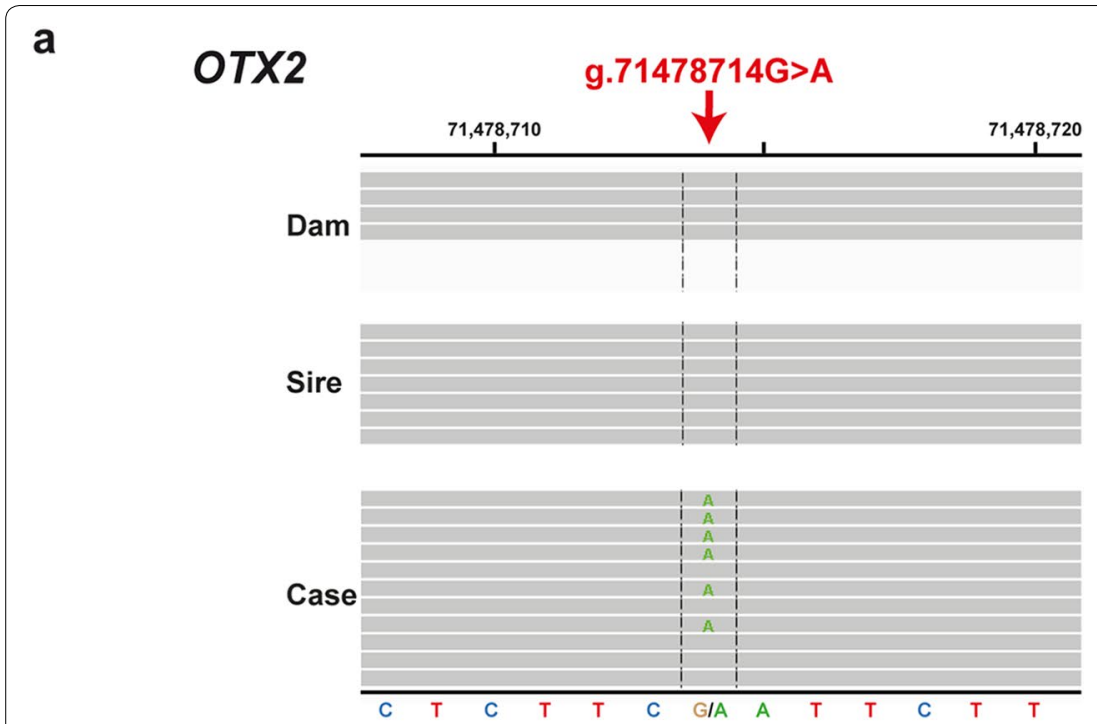

b

\section{OTX2}

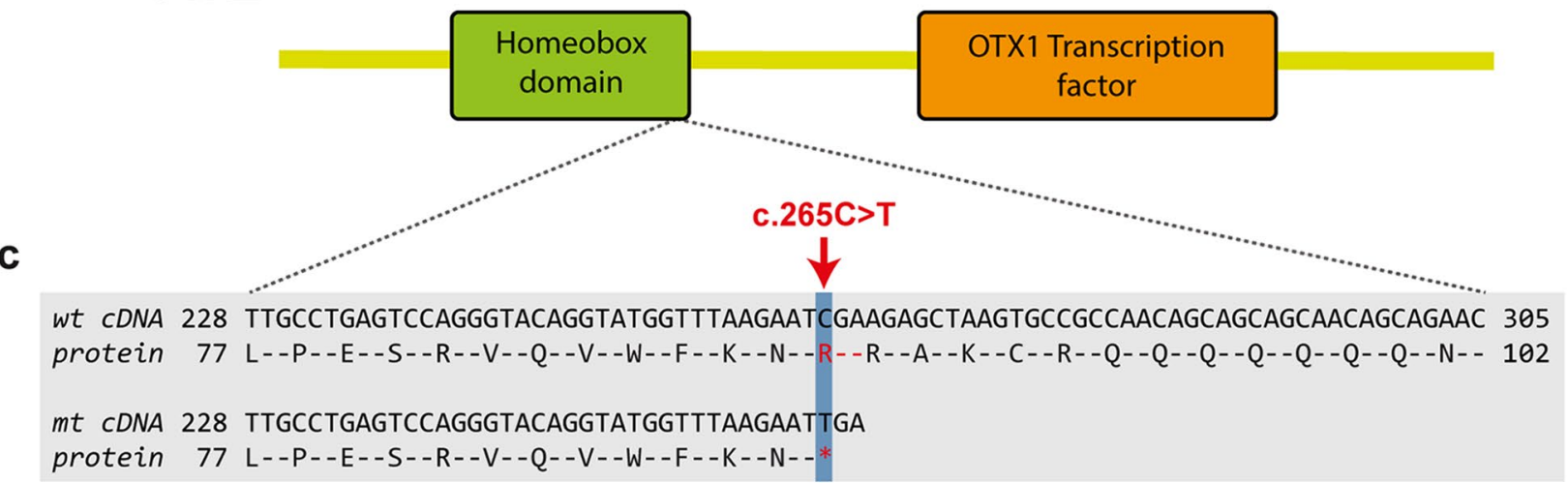

d

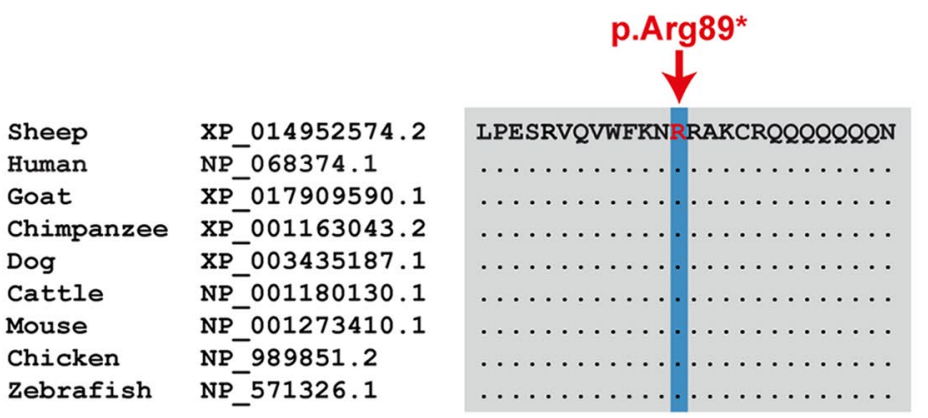

Fig. 3 Otocephaly in an Istrian Pramenka lamb—genetic analyses. a IGV (12) screenshots of the affected lamb and its parents show the missense de novo variant in the OTX2 present in the case and absent from both parents. $\mathbf{b}$ Schematic representation of the OTX2 protein showing the location of highly conserved domains. c cDNA and amino acid sequences in the region harbouring the described variant. $\mathbf{d}$ Conservation of the protein across multiple species

to represent a loss-of-function mutation, the causality of the OTX2:c.265C $>\mathrm{T}$ variant for the observed otocephaly has been established beyond a reasonable doubt. This is in accordance with the described dominantly inherited loss-of-function variants in OTX2 causing variable forms of agnathia-otocephaly complex, a pattern of malformations comprising mandibular hypoplasia/agenesis, ear anomalies, 
microstomia with oroglossal hypoplasia or aglossia, in humans (OMIM 600037) [3, 16], which were similar to the abnormalities observed in this lamb. Most of the OTX2 variants identified in patients lead to a truncated or absent protein, and thus to OTX2 haploinsufficiency, e.g. as in the case of a heterozygous OTX2 deletion associated with isolated mandibular dysostosis [17]. In a single human male fetus presented with agnathia, astomia and aglossia, absent pharyngeal floor, low posteriorly rotated, paramedian and convergent ears, a similar nonsense variant (p.Arg97*) at the corresponding position of the human OTX2 transcript was described [3].

In summary, this study reports a heterozygous de novo variant in OTX2 resulting in haploinsufficiency, in which there is no tolerance of loss-of-function variants in one copy of a gene that causes otocephaly. Recent large data from human genome sequencing studies presented in the Genome Aggregation Database (gnomAD) [18] showed that the pLI score, the probability of loss-of-function intolerance, for OTX2 is $>0.9$ meaning that OTX2 falls into the class of loss-of-function haploinsufficient genes.

This study provides an example of a pathogenic disease causing variant underlying a sporadic syndrome observed in sheep. Germline genetic variation is a quite frequent causal factor in rare diseases also in domestic animals. Therefore, collecting of samples from parents in addition to the affected offspring to perform trio-based genetic analyses is a prerequisite for successful detection of the underlying genetic cause. Precision medicine by using whole-genome sequencing has been proven suitable in recent years to help diagnose rare diseases in human patients. Progress in the field of DNA sequencing is rapidly advancing and techniques can be successfully adapted for routine diagnosis in veterinary medicine, to both confirm genetic aetiology and to obtain an insight into the molecular mechanisms involved.

\section{Supplementary information}

Supplementary information accompanies this paper at https://doi. org/10.1186/s13028-020-0503-z.

Additional file 1. List of private protein-changing sequence variants.

\section{Abbreviations}

CT: computed tomography; IGV: integrative genomics viewer; OMIA: online Mendelian inheritance in animals; OMIM: online Mendelian inheritance in man; OTX2: orthodenticle homeobox 2; PRRX1: paired related homeobox 1; SNV: single nucleotide variant; WGS: whole-genome sequencing.

\section{Acknowledgements}

The authors would like to acknowledge the Next Generation Sequencing Platform of the University of Bern for performing the whole-genome sequencing and the Interfaculty Bioinformatics Unit of the University of Bern for providing computational infrastructure.
Prior publication

Data have not been published previously.

\section{Authors' contributions}

JP and AL performed the genetic analyses and drafted the manuscript. IMH carried out the bioinformatics. TŠ and MG performed the necropsy and drafted the manuscript. AŠ and PK performed the cesarean section and collected the samples. EP performed CT analysis. CD designed the study, supervised the project and finalized the manuscript. All authors read and approved the final manuscript.

\section{Funding}

Not applicable.

\section{Availability of data and materials}

The genomic data of the sequenced sheep genomes are available under study accession no. PRJEB30931 in the European Nucleotide Archive (https:// www.ebi.ac.uk/ena; sample accessions of the studied parent-offspring trio: SAMEA5239895, SAMEA5239896 and SAMEA5239897). All references to the ovine OTX2 gene correspond to the accessions NC_040258.1 (NCBI accession), XM_015097088.2 (mRNA), and XP_014952574.2 (protein). The reference genome assembly Oar_rambouillet_v1.0 and NCBI annotation release 103 was used for WGS.

\section{Ethics approval and consent to participate}

This study did not require official or institutional ethical approval as it was not experimental. The animals were handled according to good ethical standards and Slovenian legislation. The lamb died spontaneously shortly after delivery due to the lethal character of the syndrome.

\section{Consent for publication}

Not applicable.

\section{Competing interests}

The authors declare that they have no competing interests.

\section{Author details}

${ }^{1}$ Institute of Genetics, Vetsuisse Faculty, University of Bern, Bremgartenstr. 109a, 3001 Bern, Switzerland. ${ }^{2}$ Institute of Pathology, Forensic and Administrative Veterinary Medicine, Veterinary Faculty, University of Ljubljana, Gerbičeva 60, 1000 Ljubljana, Slovenia. ${ }^{3}$ Clinic for Reproduction and Large Animals, Veterinary Faculty, University of Ljubljana, Cesta v Mestni log 47, 1000 Ljubljana, Slovenia. ${ }^{4}$ Infrastructure Centre for Sustainable Recultivation Vremščica, Veterinary Faculty, University of Ljubljana, Gabrče 30, 6224 Senožeče, Slovenia. ${ }^{5}$ Small Animal Clinic, Veterinary Faculty, University of Ljubljana, Cesta v Mestni $\log$ 47, 1000 Ljubljana, Slovenia.

Received: 31 October 2019 Accepted: 13 January 2020

Published online: 22 January 2020

References

1. Jones M, Chung J, Kimonis V, Gold JA. A novel mutation of orthodenticle homeobox 2 contributing to a case of otocephaly initially diagnosed by prenatal ultrasound in the first trimester. Clin Dysmorphol. 2017;26:98-100.

2. Chassaing N, Sorrentino S, Davis EE, Martin-Coignard D, lacovelli A, Paznekas W, et al. OTX2 mutations contribute to the otocephaly-dysgnathia complex. J Med Genet. 2012;49:373-9.

3. Patat O, van Ravenswaaij-Arts CMA. Otocephaly-dysgnathia complex: description of four cases and confirmation of the role of OTX2. Mol Syndromol. 2013;4:302-5.

4. Sergouniotis PI, Urquhart JE, Williams SG, Bhaskar SS, Black GC, Lovell SC, et al. Agnathia-otocephaly complex and asymmetric velopharyngeal insufficiency due to an in-frame duplication in OTX2. J Hum Genet. 2015;60:199-202.

5. Martínez JS, Velázquez IR, Reyes H, Fajardo R. Congenital holoprosencephaly with severe otocephaly in a Rottweiler puppy. Vet Rec. 2006;158:518-9. 
6. Brachthäuser L, Klumpp S, Hecht W, Kuchelmeister K, Reinacher M, Ebbert W, et al. Aprosencephaly with otocephaly in a lamb (Ovis aries). Vet Pathol. 2012;49:1043-8.

7. Mercer R, Steven DH. Synotia in a stillborn lamb. Vet Rec. 1966;78:94-6.

8. Pfeiffer A. Otocephalus aprosopus in the sheep. Dtsch TierarztI Wochenschr. 1968;75:407-9.

9. Shirazi A, Ahmadi E, Jadidi M, Shams-Esfandabadi N, Heidari B. Acephalous lamb from an in vitro-produced sheep embryo. Can Vet J. 2009;50:501-5

10. Glahn-Luft B, Schneider H, Schneider J, Wassmuth R. Agnathia in the sheep associated with chromosome aberration and $\mathrm{Hb}$ deficiency. Dtsch Tierarztl Wochenschr. 1978;85:472-4.

11. Chang CC, Chow CC, Tellier LCAM, Vattikuti S, Purcell SM, Lee JJ. Secondgeneration PLINK: rising to the challenge of larger and richer datasets. Gigascience. 2015;4:1-16.

12. Paris JM, Letko A, Häfliger IM, Ammann P, Flury C, Drögemüller C. Identification of two TYRP1 loss-of-function alleles in Valais Red sheep. Anim Genet. 2019;50:778-82.

13. Thorvaldsdóttir H, Robinson JT, Mesirov JP. Integrative genomics viewer (IGV): high-performance genomics data visualization and exploration. Brief Bioinform. 2013;14:178-92.
14. Lejong M, Vanmuylder N, Louryan S. External and computed tomography analysis of a strophocephalic lamb. Morphologie. 2019;103:122-5.

15. Matsuo I, Kuratani S, Kimura C, Takeda N, Aizawa S. Mouse Ot×2 functions in the formation and patterning of rostral head. Genes Dev. 1995:9:2646-58.

16. Slavotinek A. Genetics of anophthalmia and microphthalmia. Part 2: syndromes associated with anophthalmia-microphthalmia. Hum Genet 2019;138:831-46

17. Latypova X, Bordereau S, Bleriot A, Pichon O, Poulain D, Briand A, et al Mandibular dysostosis without microphthalmia caused by OTX2 deletion. Am J Med Genet A. 2016;170:2466-70.

18. Karczewski KJ, Francioli LC, Tiao G, Cummings BB, Alföldi J, Wang Q, et al. Variation across 141,456 human exomes and genomes reveals the spectrum of loss-of-function intolerance across human protein-coding genes. BioRxiv. 2019. https://doi.org/10.1101/531210v1.

\section{Publisher's Note}

Springer Nature remains neutral with regard to jurisdictional claims in published maps and institutional affiliations.
Ready to submit your research? Choose BMC and benefit from:

- fast, convenient online submission

- thorough peer review by experienced researchers in your field

- rapid publication on acceptance

- support for research data, including large and complex data types

- gold Open Access which fosters wider collaboration and increased citations

- maximum visibility for your research: over $100 \mathrm{M}$ website views per year

At BMC, research is always in progress.

Learn more biomedcentral.com/submissions 